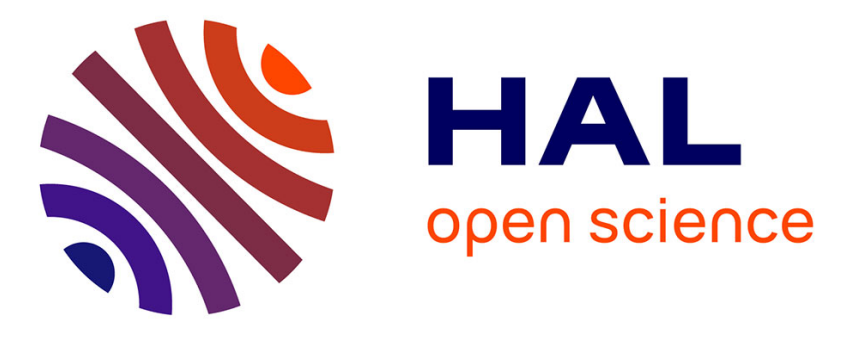

\title{
Head to pelvis alignment of adolescent idiopathic scoliosis patients both in and out of brace
}

Claudio Vergari, Isabelle Courtois, Eric Ebermeyer, Raphael Pietton, Houssam Bouloussa, Raphael Vialle, Wafa Skalli

\section{To cite this version:}

Claudio Vergari, Isabelle Courtois, Eric Ebermeyer, Raphael Pietton, Houssam Bouloussa, et al.. Head to pelvis alignment of adolescent idiopathic scoliosis patients both in and out of brace. European Spine Journal, 2019, pp.1-10. hal-02129230

\section{HAL Id: hal-02129230 \\ https://hal.science/hal-02129230}

Submitted on 14 May 2019

HAL is a multi-disciplinary open access archive for the deposit and dissemination of scientific research documents, whether they are published or not. The documents may come from teaching and research institutions in France or abroad, or from public or private research centers.
L'archive ouverte pluridisciplinaire HAL, est destinée au dépôt et à la diffusion de documents scientifiques de niveau recherche, publiés ou non, émanant des établissements d'enseignement et de recherche français ou étrangers, des laboratoires publics ou privés. 


\title{
Head to pelvis alignment of adolescent idiopathic scoliosis patients in and out of brace
}

\author{
C.Vergari $^{\mathrm{a}}$, I.Courtois ${ }^{\mathrm{b}}$, E.Ebermeyer ${ }^{\mathrm{b}}$, R.Pietton ${ }^{\mathrm{c}}$, H.Bouloussa ${ }^{\mathrm{c}, \mathrm{d}}$, \\ R.Vialle ${ }^{c}$, W.Skalli ${ }^{\mathrm{a}}$ \\ ${ }^{a}$ Arts et Metiers ParisTech, LBM/Institut de Biomecanique Humaine Georges Charpak, \\ Paris, France. \\ ${ }^{b}$ Unite Rachis, CHU - Hopital Bellevue, Saint-Etienne, France \\ ${ }^{c}$ Department of Paediatric Orthopaedics, Armand Trousseau Hospital, Université Pierre \\ et Marie Curie-Paris 6, Paris, France \\ $d$ \\ Kaiser Oakland Medical Center, Spine Surgery Department, CA 94611, United States
}

\begin{abstract}
Purpose To determine the short-term effect of bracing of adolescent idiopathic scoliotic (AIS) patients on the relationships between spinopelvic parameters related to balance, by comparing their in and out-of-brace geometry and versus healthy subjects.

Methods Forty-two AIS patients (Cobb angle $29^{\circ} \pm 12^{\circ}$, ranging from 16 to $61^{\circ}$ ) with a prescription of orthotic treatment were included retrospectively and prospectively. They all underwent biplanar radiography and $3 \mathrm{D}$ reconstruction of the spine and pelvis before bracing as well as less than 9 months after bracing. Eighty-three age-matched healthy adolescents were also included as control group and underwent biplanar radiography and 3D reconstruction.

Results Sacral slope was higher in AIS than healthy patients $(\mathrm{p}=0.005)$. Bracing induced large changes of pelvic tilt (between $-9^{\circ}$ and $9^{\circ}$ ), although patients' sagittal spinopelvic alignment tended to remain within the normality corridors defined by the healthy patients. Patients had flatter backs compared to healthy subjects and bracing further reduced their spinal curves. The head tended to remain above the pelvis in-brace.

Conclusions Analysis of sagittal alignment from head to pelvis showed that bracing further flattened the patients' backs and induced large compensating reorientations of the pelvis. Sagittal balance should be included in the planning and evaluation of brace treatment, since it could play a role in its outcome.
\end{abstract}

Keywords: AIS; bracing, brace effect, sagittal balance, compensation 


\section{Introduction}

Scoliosis is a complex deformity of the trunk in the three planes of space. Its most prominent characteristic is a deviation of the spinal curve in the coronal plane, which is usually quantified by the Cobb angle, together with a flattening of the sagittal spinal curve and vertebral rotations in the transverse plane. Currently, the attention is shifting from a $2 \mathrm{D}$ characterisation of the deformity through the Cobb angle, towards a global 3D analysis of the patient, including their alignment. This should ideally include the spinal chain of balance from the head to the "pelvic vertebra" and, in some cases, the lower limbs [1].

Patients tend to compensate any alteration of the balance in order to keep the head upon the pelvis and a horizontal gaze: the compensation is obtained by altering the spinal alignment and, for congruity, pelvis orientation [2]. When spinal flexibility is reduced or pelvic reorientation does not suffice to keep the head upon the pelvis, such as in elderly patients, lower limbs are the last link of the compensatory chain that can help them maintain their balance [3].

Analysis of spinopelvic parameters and their mutual relationships have been thoroughly characterized in healthy adolescents [4] and adults [5-9]. In particular, the interest of patient balance is recognized for spinal surgery [10, 11], which is the final recourse to correct progressive scoliotic patients for whom the non-operative treatment failed. Post-surgery malalignment can be a source of pain and disability, and it can lead to severe mechanical consequences such as proximal junctional failure or adjacent disc degeneration [12-15].

Adolescent idiopathic scoliosis (AIS) is the most common form of the pathology, with risk of progression especially during the pubertal growth spurt. The most common conservative treatment for progressive moderate to severe curves is bracing, which aims to stop or slow down the progression to avoid surgery. Just like the assessment of scoliosis severity, evaluation of brace effect on the spinal alignment of AIS patients is often only focused on Cobb angle correction. Thanks to relatively recent technical advancements in 3D imaging of scoliosis in clinical routine[16], three-dimensional parameters started being included in the analysis of bracing [17-19]. This shows that bracing can have limited effects on the 3D deformity: it can leave the thoracic

kyphosis and axial rotations unchanged or even worsened in up to $80 \%$ of cases [17]. This shows how the correction mechanisms of braces are still not 
completely understood. Several factors account for this: the complex shape of the deformity, the difficulty of planning and implementing an effective correction strategy, but also the compensating mechanisms that are usually not accounted for when planning a brace.

To our knowledge, no study analysed the effects of bracing on the relationships between spinopelvic parameters, rather than on the parameters themselves. The hypothesis of this work is that such analysis could clarify how bracing acts on the deformity and give an insight on the compensation mechanisms of young AIS patients.

\section{Materials and Methods}

\subsection{Subjects}

Forty-two patients ( 27 girls and 15 boys, average age $13 \pm 2$, average Cobb angle $29^{\circ} \pm 12^{\circ}$, ranging from 16 to $61^{\circ}$ ) diagnosed with progressive AIS were included retrospectively and prospectively from 2 clinical centres. Patients were treated if they presented a Risser sign $\leq 2$, Cobb angle $\geq 25^{\circ}$ [20] or an increase of more than $5^{\circ}$ in 6 months paired with a worsening of the clinical appearance with hump increase [21]. Twenty-three patients had thoracic curves (apex in T1-T11), nine were thoracolumbar (apex in T12-L1) and ten were lumbar (apex in L2-L5). All patients were at their first prescription of treatment; 30 patients wore thoracic lumbar sacral orthosis (TLSO) and 12 night-time braces. All patients underwent biplanar radiographs (EOS system, EOS Imaging, Paris, France) in free-standing position at treatment decision (pre-brace) and in-brace. In-brace acquisition was performed on average $3 \pm 2$ months after pre-brace x-ray (ranging between same day and 9 months).

Eighty-three healthy adolescents and young adults (58 girls, 25 boys, mean age $14 \pm 3$ years, ranging between 9 and 21 years) were also included as control group. They all underwent biplanar radiography. The study was performed after approval of the ethical committee (CPP 6001 Ile de France $\mathrm{V}$ and local hospital committee).

\subsection{Data processing and analysis}

Validated methods were employed to reconstruct the 3D geometry of the spine and pelvis for each biplanar X-ray [22]. In addition, the odontoid process $(\mathrm{OD})$ and the bicoxo-femoral axis were digitized in both radiographs (Figure 1). An anatomical coordinate system was calculated for each patient, 

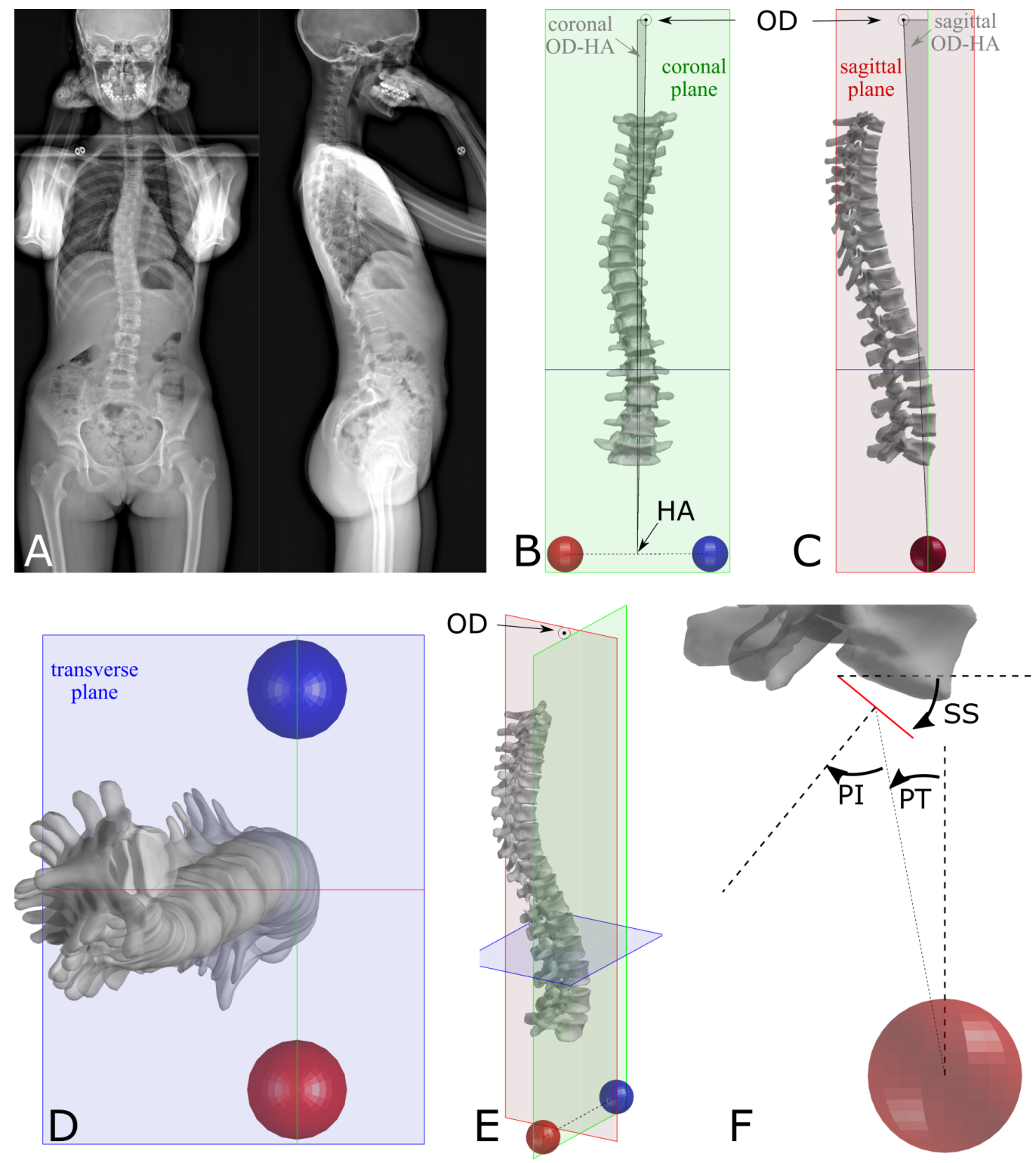

Figure 1: Biplanar radiographs of an adolescent idiopathic scoliotic patient (A) and 3D reconstruction of the pelvis, spine and $\mathrm{C} 2$ odontoid process (OD) in frontal (B), sagittal (C), top (D) and 3D views. Panel B reports the coronal projection of the OD-HA angle and panel $\mathrm{C}$ the sagittal one. Panel $\mathrm{F}$ represents the pelvic parameters (pelvic tilt: PT, pelvic incidence: PI, sacral slope: SS) and the positive sign of each parameter is indicated by the arrows. 
with its origin placed at the centre of the bicoxo-femoral axis (HA). The coronal plane was considered vertical and passing through the two acetabula, the sagittal plane was vertical and at $90^{\circ}$ from the coronal plane, while the transverse plane was horizontal.

Clinical parameters were calculated in the patient coordinate system from the 3D reconstruction: pelvic incidence $(\mathrm{PI})$, pelvic tilt $(\mathrm{PT})$, sacral slope (SS), L1-S1 lumbar lordosis (LL), T1-T12 thoracic kyphosis (TK). In addition, the angle between the vertical and the line that connects OD to HA was measured in the sagittal and coronal planes (Figure 1, [23]).

Vialle et al. and Schwab et al. previously reported several significant relationships between pelvic and spinal parameters in healthy subjects $[6,9]$ (SS vs PI, PT vs PI, LL vs PI, LL vs a linear combination of PT, PI and TK). For each of these relationships, a confidence ellipse was built [24] using the data from the previous work to represent the distribution of the different parameters in the healthy population and compare it with the present data on healthy subjects and AIS patients.

\subsection{Statistics}

Non-parametric tests were selected because not all variables passed normality tests (Lilliefors' test). Differences between pre-brace and in-brace parameters were analysed with paired Wilcoxon signed rank test, while comparisons with healthy controls using Kruskal Wallis test and Tukey-Kramer post-hoc analysis. Correlations were quantified with Spearman's rank test. Slopes and intercepts of the linear regression were compared between groups using rank transform analysis of covariance (ANCOVA) [25]. Analyses were performed with Matlab 2016b (The Mathworks, Natick, MA, USA).

\section{Results}

Age and sex distributions were not significantly different between healthy and AIS subjects $(\mathrm{p}>0.05)$. Table 1 reports the clinical parameters for the healthy subjects and the AIS patients before and in brace. Curve topology had no significant impact on brace effect $(\mathrm{p}>0.05$, Kruskal-Wallis test).

\subsection{Pelvis}

Figure 2 shows the relationship between PI and SS in each group, compared to the normal distribution published by Vialle et al. [9]. It can be 

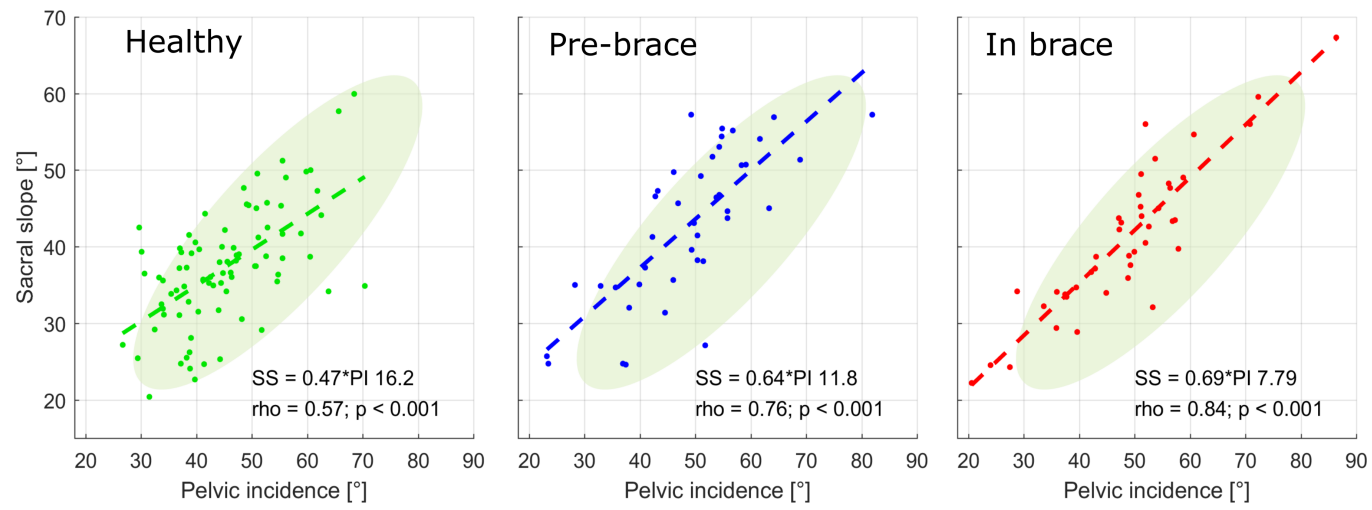

Figure 2: Relationship between pelvic incidence and sacral slope in healthy subjects, prebrace and in-brace scoliotic patients. Dashed lines represent linear regressions (equations and correlation coefficients are reported in each panel) while shaded areas represent the $95 \%$ confidence ellipse of healthy subjects from Vialle et al. [9].

noted that the data for pre-brace and in-brace patients appears shifted upwards relative to the normal ellipse; indeed, SS was higher in AIS patients pre-brace than healthy subjects $(\mathrm{p}=0.005)$. PT and linear regressions did not differ between groups $(\mathrm{p}>0.05)$.

Figure 3 shows the relationship between PI and PT in each group. Again, regression parameters did not statistically vary between groups. PT was lower in AIS patients than healthy subjects, but the difference was not significant, nor was brace effect significant $(\mathrm{p}=0.37$, Table 1$)$. Nevertheless, PT change due to bracing ranged from $-9^{\circ}$ to $9^{\circ}$; in other words, while the average PT did not change between pre-brace and in-brace, large PT variations took place. Further analysis showed that all patients (but one) who had a pelvic tilt lower than $5^{\circ}$ increased their pelvic tilt when in-brace, while $64 \%$ of the patients who had pelvic tilt higher than $5^{\circ}$ decreased it (Figure $4)$.

\subsection{Spinal Alignement}

Figure 5 shows the relationship between PI and LL; the intercept of the linear regression for healthy subjects was significantly higher than in the other groups $(\mathrm{p}<0.001)$, while the slope was lower but not significantly. In other words, for a given SS, AIS patients tended to have flatter lordotic spines (because LL values are negative). Moreover, AIS patients further decreased their lordosis when in brace $(\mathrm{p}=0.04$, Table 1$)$. The change in LL was 

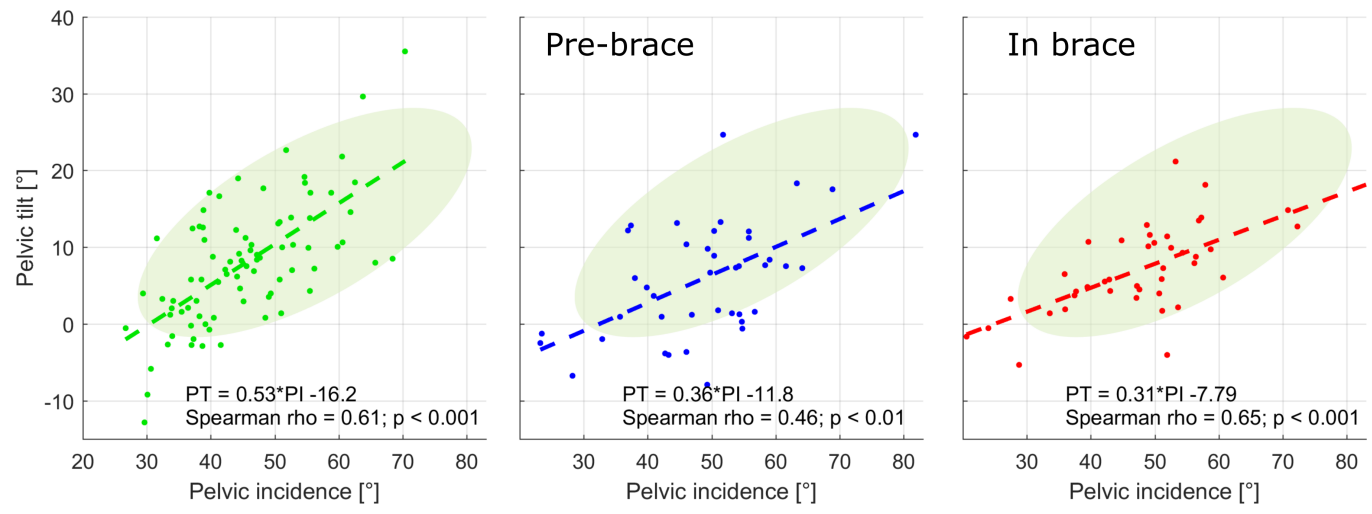

Figure 3: Relationship between pelvic incidence and pelvic tilt in healthy subjects, prebrace and in-brace scoliotic patients. Dashed lines represent linear regressions (equations and correlation coefficients are reported in each panel) while shaded areas represent the $95 \%$ confidence ellipse of healthy subjects from Vialle et al. [9].
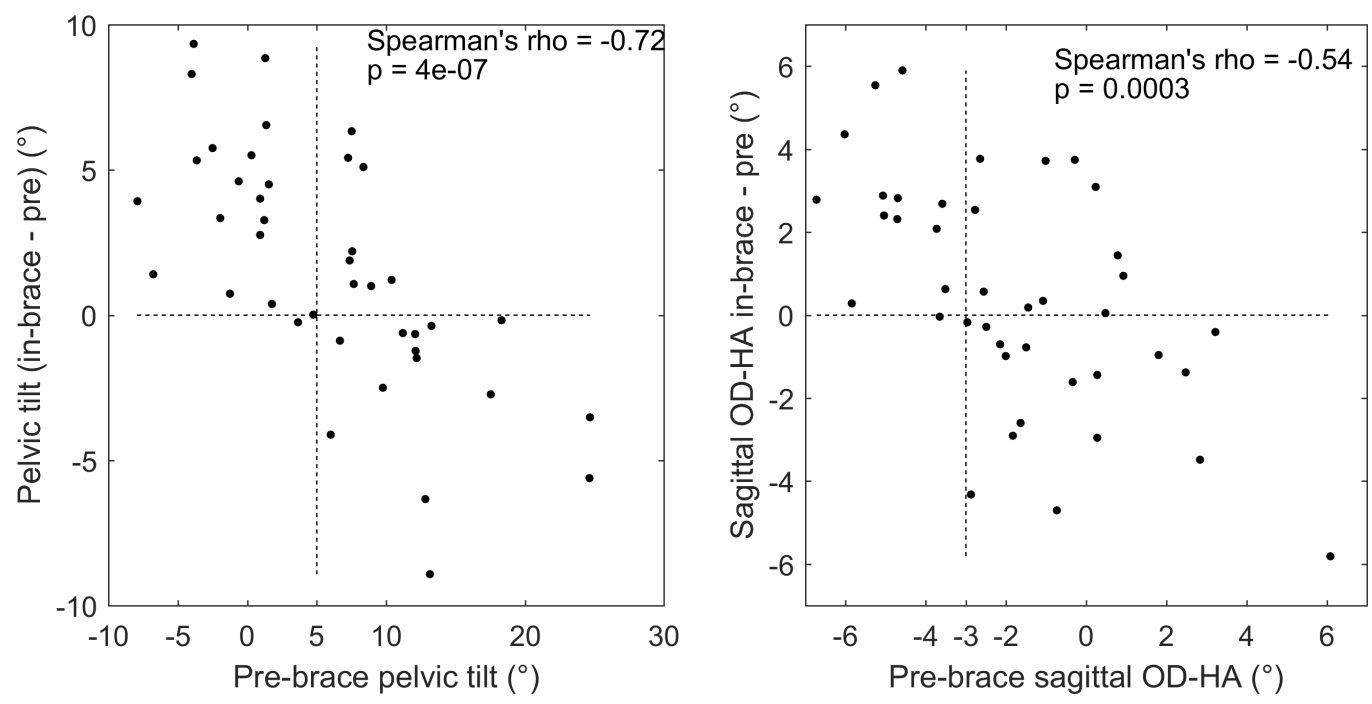

Figure 4: Difference between in-brace and pre-brace pelvic tilt and OD-HA sagittal angle relative to pre-brace values. All patients but one who had a pelvic tilt lower than $5^{\circ}$ increased their pelvic tilt when in-brace, while $64 \%$ of the patients who had pelvic tilt higher than $5^{\circ}$ decreased it. Similarly, all patients but one who had sagittal OD-HA lower than $-3^{\circ}$ increased their OD-HA in brace, while $61 \%$ of the patients who had OD-HA higher than $-3^{\circ}$ decreased it 
Table 1: Clinical parameters in healthy subjects and adolescent idiopathic scoliosis patients before and in-brace.

\begin{tabular}{|c|c|c|c|c|}
\hline & $\begin{array}{l}\text { Healthy } \\
\text { subjects }\end{array}$ & Pre-brace & In-brace & $\begin{array}{l}\text { Difference } \\
\text { between groups }\end{array}$ \\
\hline $\begin{array}{l}\text { Cobb } \\
\text { angle }\left[{ }^{\circ}\right]\end{array}$ & - & $29.2 \pm 12.1$ & $19.1 \pm 9.8$ & In-brace $<$ pre-brace $(\mathrm{p}=0)$ \\
\hline $\begin{array}{l}\text { T1-T12 } \\
\text { kyphosis }\left[{ }^{\circ}\right]\end{array}$ & $40.6 \pm 10.9$ & $34.2 \pm 15.7$ & $31.0 \pm 14.4$ & $\begin{array}{l}\text { Healthy }>\text { pre-brace }(\mathrm{p}=0.02) \\
\text { Healthy }>\text { in-brace }(\mathrm{p}=0.0004)\end{array}$ \\
\hline $\begin{array}{l}\text { L1-S1 } \\
\text { Lordosis }\left[{ }^{\circ}\right]\end{array}$ & $-54.3 \pm 11.1$ & $-58.2 \pm 12.5$ & $-52.5 \pm 10.8$ & In-brace $>$ pre-brace $(\mathrm{p}=0.04)$ \\
\hline $\begin{array}{l}\text { Pelvic } \\
\text { incidence }\left[{ }^{\circ}\right]\end{array}$ & $45.3 \pm 9.8$ & $48.8 \pm 11.7$ & $48.3 \pm 12.6$ & $\mathrm{p}=0.09$ \\
\hline $\begin{array}{l}\text { Pelvic } \\
\text { tilt }\left[{ }^{\circ}\right]\end{array}$ & $7.9 \pm 8.0$ & $6.0 \pm 7.8$ & $7.3 \pm 5.8$ & $\mathrm{p}=0.37$ \\
\hline $\begin{array}{l}\text { Sacral } \\
\text { slope }\left[{ }^{\circ}\right]\end{array}$ & $37.4 \pm 7.6$ & $42.9 \pm 9.9$ & $41.0 \pm 9.7$ & Healthy $<$ pre-brace $(\mathrm{p}=0.005)$ \\
\hline
\end{tabular}

significantly correlated to the change in PT (Spearman's rho $=0.48, \mathrm{p}=$ $0.001)$.

T1-T12 kyphosis was significantly higher in healthy patients that in prebrace and in-brace (Table 2). Figure 6 shows a multilinear relationship between LL vs a combination of PT, PI and TK, as previously described by Vialle et al. [9]. This relationship, which includes the thoracolumbar spine and pelvis, was similar between groups, even if LL and TK showed differences.

\subsection{Odontoid process}

Figure 7 reports OD-HA sagittal and coronal angle in the three groups. Both sagittal and coronal angles tended to increase from healthy to pre-brace and to in-brace, although differences between groups were not significant. Similarly to brace-induced changes in PT, changes in sagittal OD-HA were not statistically significant but they ranged between $-6^{\circ}$ and $+6^{\circ}$. Again, changes depended on the pre-brace angle (Figure 4).

\section{Discussion}

This work focused on the analysis of spinopelvic alignment in AIS patients at decision of conservative treatment and in-brace, compared to healthy subjects. Only the immediate effects of bracing were analysed, 3 months after 

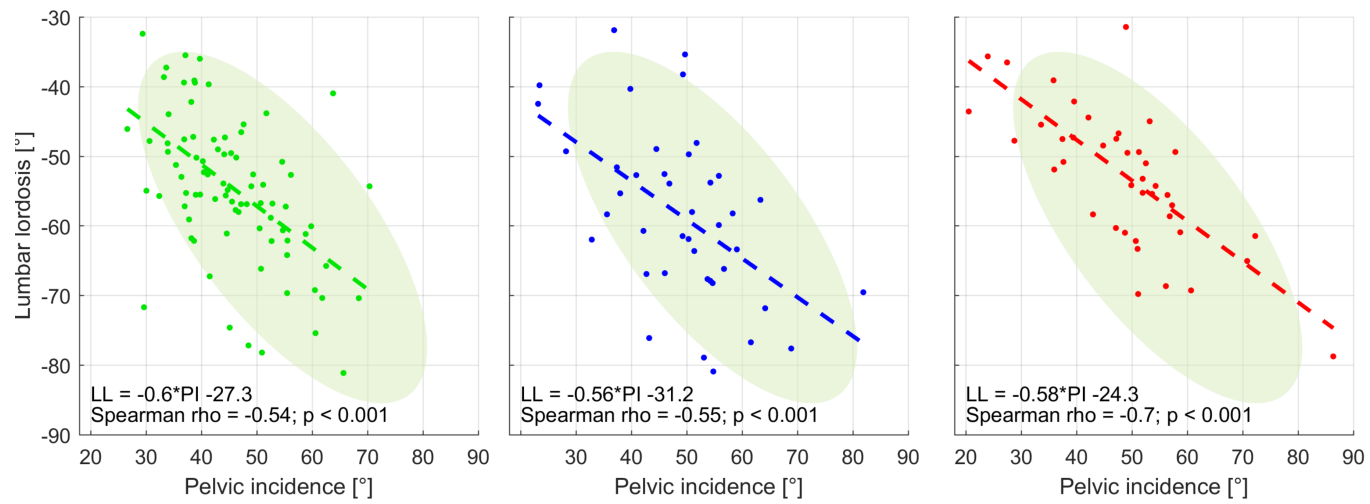

Figure 5: Relationship between pelvic incidence and L1-L5 lumbar lordosis in healthy subjects, pre-brace and in-brace scoliotic patients. Dashed lines represent linear regressions (equations and correlation coefficients are reported in each panel) while shaded areas represent the $95 \%$ confidence ellipse of healthy subjects from Vialle et al. [9].
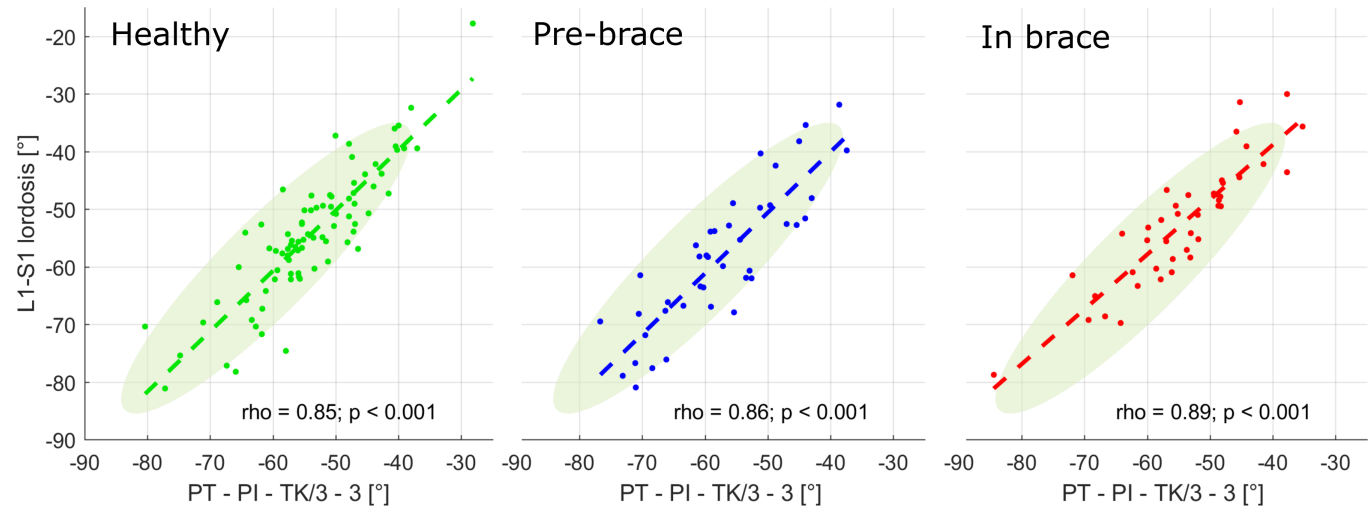

Figure 6: Relationship between L1-S1 lordosis, Pelvic tilt (PT), pelvic incidence (PI) and T1-T1 thoracic kyphosis (TK) in healthy subjects, pre-brace and in-brace scoliotic patients. Dashed lines represent linear regressions (correlation coefficients and p-values are reported in each panel) while shaded areas represent the $95 \%$ confidence ellipse of healthy subjects from Vialle et al. [9].

the last out-of-brace biplanar radiography. While short-term effect cannot be used to determine brace efficacy for the long-term outcome, several studies suggest that the two are correlated, thus confirming the interest in analysing immediate brace effects $[20,26,27]$. The originality of this work lies in the analysis of the relationship between parameters, compared to those obtained in healthy patients, in addition to the range and distribution of each single 
parameter. This approach allows an insight into the strategy to maintain balance of AIS patients relative to healthy subjects, and how this strategy could be affected by bracing.

While the analysis was mainly focused on sagittal plane parameters, these were calculated from a 3D model. This allows correction of projection biases; for instance, if the patient was not perfectly aligned with the lateral plane of the radiographic device, their pelvic parameters would still be calculated in the anatomical sagittal plane rather than in the radiographic plane. The $3 \mathrm{D}$ geometry of the spine is also accounted for when calculating sagittal parameters, which are classically measured in 2D, since they can be measured using the $3 \mathrm{D}$ orientation of the vertebral endplates.

Results were compared with $95 \%$ confidence ellipses calculated on the data reported by Vialle et al. on a population of healthy subjects aged from 20 to 70 years

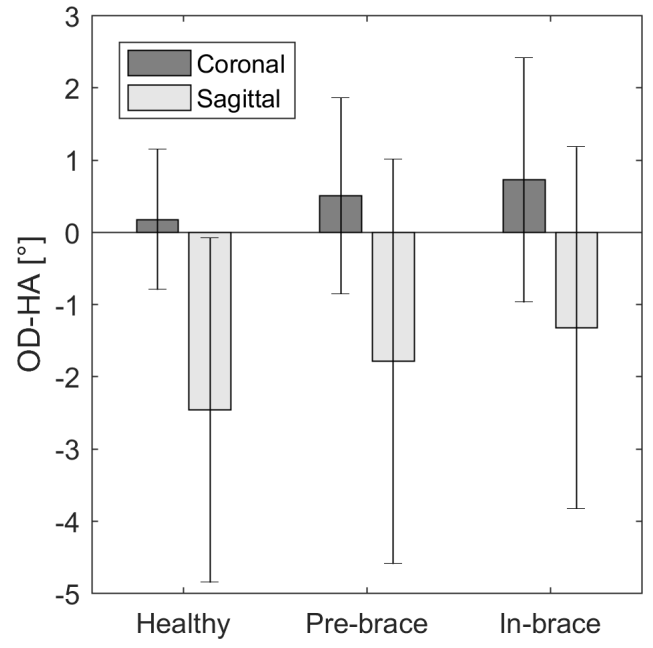

Figure 7: OD-HA angle in the coronal and sagittal planes for the three groups. Differences between groups were not significant. old [9]. Since the age range in the present study was limited to adolescents, it was necessary to include a population of healthy adolescents to determine if the previously published spinopelvic relationships remained valid. Figures 3- 4 and 5- 6 show that this is the case; only PI appeared smaller in this study (45.3 $\pm 9.8^{\circ}$ against $54.7 \pm 10.6^{\circ}$ in Vialle et al.). This could be expected, since PI tends to increase with age.

The advantage of comparing patient clinical parameters with $95 \%$ confidence ellipses based on healthy subjects, is that ellipses simultaneously represent the distribution of data in two dimensions, the slope of the relationship between parameters and the correlation coefficient of the underlying linear regression (represented by the transversal diameter of the ellipse). In other words, there is an immediate comparison with the linear regression between parameters of healthy patients and with the parameters' confidence intervals.

Spinal sagittal alignment was further flattened by bracing: kyphosis was lower in AIS than in healthy subjects, as expected, but it was further reduced by bracing. Lordosis was also reduced by bracing, thus worsening the 
characteristic scoliotic "flat back", confirming previous 3D analyses of brace effect [17]. The general consensus is that bracing should aim at restoring a normal 3D geometry of the trunk, without neglecting the transverse and sagittal planes. These were included in the SOSORT guidelines for scoliosis treatment [24, 25]. Nevertheless, brace is still often prescribed only using a coronal x-ray, thus neglecting the 3D configuration. Figure 6 shows a multilinear relationship representing a global spino-pelvic alignment, since it includes PT, PI, TK and LL. Even though TK and LL differences were present between groups (for instance, in TK and LL, Table 1), this graph remains within the confidence ellipse for the three groups, suggesting that global sagittal alignment remained balanced even after the loss of kyphosis and lordosis.

Bracing had variable effect on PT, with some patients increasing and other decreasing it (Figure 4), and the change in PT was significantly correlated to the change in LL $(\mathrm{p}=0.001)$. Thus, patients who flattened their LL in brace, compensated by increase their PT (pelvic retroversion). This is expected, but a non-negligible portion of patients $(\mathrm{N}=10)$ actually decreased their PT while also decreasing LL. Figure 8 and Online Resource 1 show such a case; this patient had a high pre-brace PT $\left(13^{\circ}, 85\right.$ th percentile $)$, but her head was posterior relative to her acetabula $\left(\mathrm{OD}-\mathrm{HA}=-5^{\circ}\right)$. Her strategy to compensate the sagittal straightening of her spine was to pivot her whole trunk forward around her pelvic acetabula, resulting in a forward shift of the head, which actually brought the head close to the vertical of the acetabula (in-brace OD HA $=1.3^{\circ}$ ).

As shown by the description of this compensation mechanism, the inclusion of the head in the analysis of brace effect is an original aspect of this work. OD is just below the head's centre of mass [29], and it has the advantage over the auditory meatuses of being easily recognizable in frontal and lateral radiographs. It is commonly accepted that all subjects tend to maintain the head upon the pelvis and a horizontal gaze, with minimal expenditure of energy $[2,30]$. This is because the head has a non-negligible weight which is applied to the relatively small cervical spine; a small displacement of the head could induce higher muscular forces to counterbalance large moments on the cervical vertebrae and discs. Indeed, recent studies showed that the OD-HA angles are almost constant in asymptomatic subjects, whether young or elderly [31].

This result was confirmed in AIS patients, who showed the same variability of sagittal OD-HA (SD: $2.8^{\circ}$, Table 2 ) as healthy subjects in the 


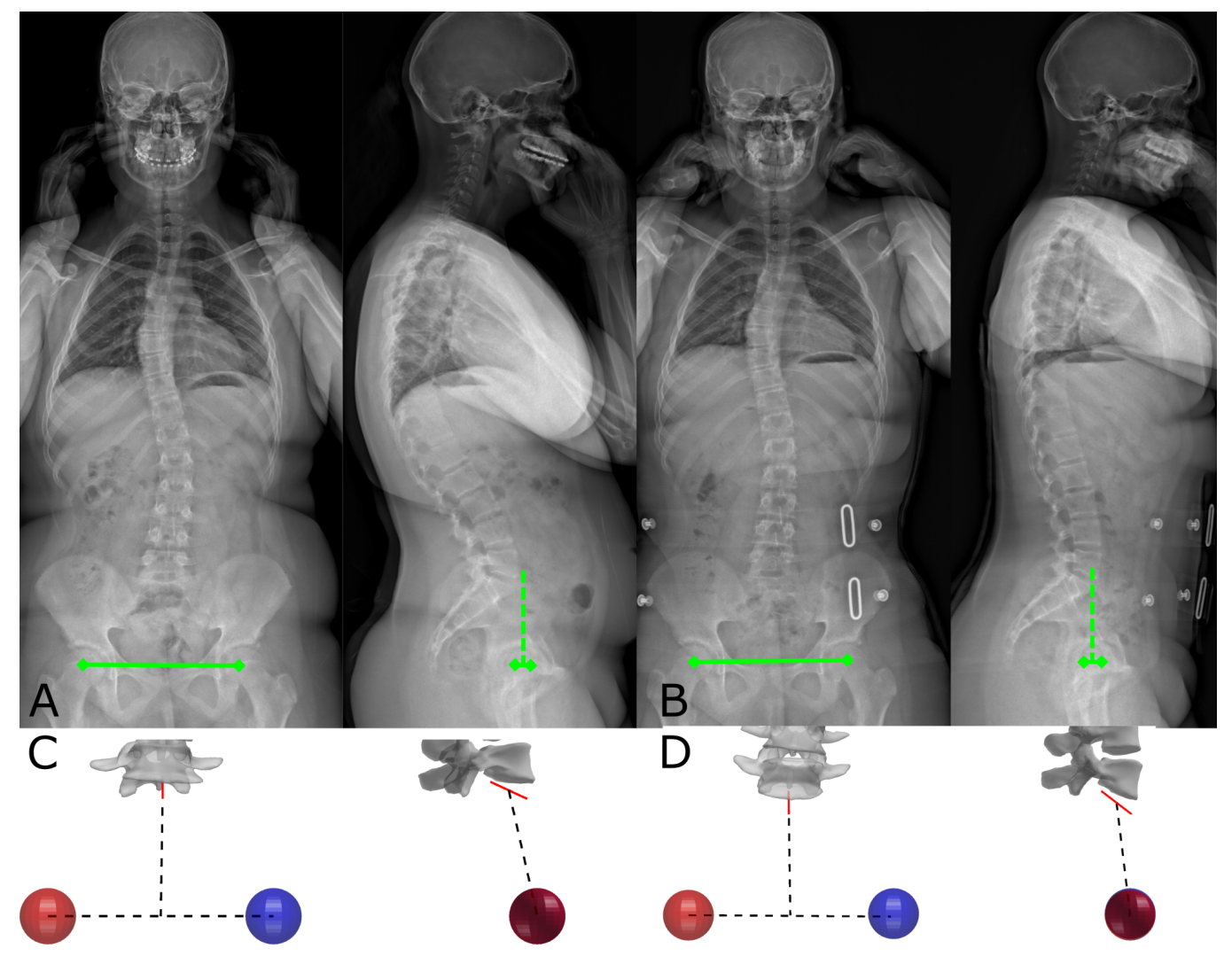

Figure 8: Biplanar radiographies of a patient before (A) and in brace (B). Green lines show the interacetabular axis and the vertical line from its midpoint. This patient, who had a pelvic incidence of $37^{\circ}$, significantly flattened her lumbar lordosis in brace (from $-52^{\circ}$ to $-39^{\circ}$ ) while reducing her pelvic tilt (from $13^{\circ}$ to $7^{\circ}$ ). $3 \mathrm{D}$ reconstruction of the pelvis $(\mathrm{C}-\mathrm{D}$ ) shows the forward shift of the sacral plate in brace (D), i.e. the pelvic anteversion.

present work (SD: $2.4^{\circ}$ ) and in the previous study from Amabile et al. (SD: $2^{\circ}$ ). Bracing did not affect variability (SD: 2.5), and the same applied for the coronal angle, which presented even lower variability (less than $2^{\circ}$ in all groups). Nevertheless, AIS patients in-brace showed a small forward shift of the head relative to healthy subjects $\left(-1.3^{\circ}\right.$ sagittal OD-HA against $-2.5^{\circ}$, respectively), corresponding to a $1 \mathrm{~cm}$ displacement (Table 2). This could be due to the reduced kyphosis which were compensated by a forward shift of the body inclination.

Lower limbs were not included in the analysis of sagittal balance, but it is generally accepted that they mostly intervene in compensation mechanisms 
of elderly subjects, who present less spinal and pelvic mobility, or adolescents with high-grade spondylolisthesis. A further limitation of this study is that the cohort was not sufficiently large to perform a sub-analysis concerning the different effects of night-time and TLSO braces, although this was beyond the scope of this work. Finally, patients were recruited from only two centres, and therefore results should be generalized with care. Type and quality of bracing depends on the orthotist's experience, and different centres could have different approaches to brace manufacturing: a larger multi-centric cohort could increase result variability. Quantitative analysis of the sagittal plane is important in bracing, because its correction could play a role in treatment outcome. For instance, it was previously suggested that negative pre-brace pelvic tilt could be indicative of curve progression [32]. In the present study, brace-induced changes of PT and OD-HA depended on the out-of-brace values. Patients who had low out-of-brace PT tended to increase them proportionally, and vice-versa; the same principle applied to the position of the head (Figure 4). In other words, patients tended to normalise their pelvic orientation and the position of the head above the pelvis. This observation gives an insight on the compensation strategy actuated by the patient, although this strategy remains a complex combination of neuromuscular activity, dynamic adaptation, proprioception and biomechanics, which cannot be summed up in simplified rules than will be true for all patients.

\section{Conclusion}

Bracing enabled a significant correction of Cobb angle, but analysis of sagittal alignment from head to pelvis showed that it further flattened the patients' backs. This resulted in large compensating reorientations of the pelvis to keep the congruity with spinal alignment and to keep maintain the head upon the pelvis. Sagittal balance should be included in the planning and evaluation of bracing treatment. Indeed, its systematic measurement could play a role in treatment outcome and, considering the present analysis, help improve brace action on the sagittal deformity.

\section{Acknowledgements}

The authors are grateful to the BiomecAM chair program on subjectspecific musculoskeletal modelling (with the support of ParisTech and Yves Cotrel Foundations, Société Générale, Covea and Proteor) and to the DHU 
MAMUTH for funding. We are also grateful to Ms Fay Manning for her technical support.

\section{References}

1. Dubousset J (2018) Definition of Adolescent Idiopathic Scoliosis Pathogenesis of Idiopathic Scoliosis. In: Machida M, Weinstein SL, Dubousset J (eds). Springer Japan, Tokyo, pp 1-25

2. Le Huec JC, Gille O, Fabre T (2018) Sagittal balance and spine-pelvis relation: A French speciality? Orthop Traumatol Surg Res 104:551-554. https://doi.org/10.1016/j.otsr.2018.06.001

3. Barrey C, Roussouly P, Le Huec JC, et al (2013) Compensatory mechanisms contributing to keep the sagittal balance of the spine. Eur Spine J 22:834-841. https://doi .org/10.1007/s00586-013-3030-z

4. Vedantam R, Lenke LG, Keeney JA, Bridwell KH (1998) Comparison of Standing Sagittal Spinal Alignment in Asymptomatic Adolescents and Adults. Spine (Phila Pa 1976) 23:

5. Le Huec JC, Hasegawa K (2016) Normative values for the spine shape parameters using 3D standing analysis from a database of 268 asymptomatic Caucasian and Japanese subjects. Eur Spine J 25:3630-3637. https ://doi. org/10.1007/s00586-016-4485-5

6. Schwab F, Lafage V, Patel A, Farcy JP (2009) Sagittal plane considerations and the pelvis in the adult patient. Spine (Phila Pa 1976) 34:1828-1833. https://doi.org/10.1097/BRS.0b013e3181a13c08

7. Legaye J, Duval-Beaupère G (2005) Sagittal plane alignment of the spine and gravity a radiological and clinical evaluation. Acta Orthop Belg 71:213-220. https://doi.org/10.1016/j.nec.2007.02.008

8. Vaz G, Roussouly P, Berthonnaud E, Dimnet J (2002) Sagittal morphology and equilibrium of pelvis and spine. Eur Spine J 11:80-87. https: //doi.org/10.1007/s005860000224

9. Vialle R, Levassor N, Rillardon L, et al (2005) Radiographic Analysis of the Sagittal Alignment and Balance of the Spine in Asymptomatic Subjects. J Bone Jt Surg 87:260-267. https://doi .org/10.2106/JBJS.D.02043

10. Lafage V, Schwab F, Vira S, et al (2011) Spino-Pelvic Parameters After Surgery Can be Predicted: A Preliminary Formula and Validation of Standing Alignment. Spine (Phila Pa 1976) 36:

11. Roussouly P, Labelle H, Rouissi J, Bodin A (2013) Pre- and postoperative sagittal balance in idiopathic scoliosis: a comparison over the ages 
of two cohorts of 132 adolescents and 52 adults. Eur Spine J 22:203-215. https://doi.org/10.1007/s00586-012-2571-x

12. A. Alzakri, C. Vergar, M. Van den Abbeele, O. Gille, W. Skalli, I. Obeid (2019) Global Sagittal Alignment and Proximal Junctional Kyphosis in Adolescent Idiopathic Scoliosis. Spine Deformity 7(2): 236-244

13. Glassman SD, Bridwell K, Dimar JR, et al (2005) The Impact of Positive Sagittal Balance in Adult Spinal Deformity. Spine (Phila Pa 1976) 30:

14. Lazennec J-Y, Ramaré S, Arafati N, et al (2000) Sagittal alignment in lumbosacral fusion: relations between radiological parameters and pain. Eur Spine J 9:47-55. https : //doi .org/10.1007/s005860050008

15. Kumar M, Baklanov A, Chopin D (2001) Correlation between sagittal plane changes and adjacent segment degeneration following lumbar spine fusion. Eur Spine J 10:314-319. https://doi.org/10.1007/s005860000239

16. Dubousset J, Charpak G, Dorion I, et al (2005) [A new 2D and 3D imaging approach to musculoskeletal physiology and pathology with low-dose radiation and the standing position: the EOS system]. Bull Acad Natl Med 189:287-300

17. Courvoisier A, Drevelle X, Vialle R, et al (2013) 3D analysis of brace treatment in idiopathic scoliosis. Eur Spine J 22:2449-2455. https: //doi.org/10.1007/s00586-013-2881-7

18. Lebel DE, Al-Aubaidi Z, Shin E-J, et al (2013) Three dimensional analysis of brace biomechanical efficacy for patients with AIS. Eur Spine J 22:2445-2448. https://doi.org/10.1007/s00586-013-2921-3

19. Clin J, Aubin C-E, Parent S, et al (2010) Comparison of the biomechanical 3D efficiency of different brace designs for the treatment of scoliosis using a finite element model. Eur Spine J 19:1169-1178. https: //doi .org/10.1007/s00586-009-1268-2

20. Negrini S, Donzelli S, Aulisa AG, et al (2018) 2016 SOSORT guidelines: orthopaedic and rehabilitation treatment of idiopathic scoliosis during growth. Scoliosis Spinal Disord 13:3.

https://doi.org/10.1186/s13013-017-0145-8

21. Lonstein JE, Carlson JM (1984) The prediction of curve progression in untreated idiopathic scoliosis during growth. J Bone Jt Surg - Am Vol 66:1061-1071

22. Humbert L, De Guise JA, Aubert B, et al (2009) 3D reconstruction of the spine from biplanar X-rays using parametric models based on transversal and longitudinal inferences. Med Eng Phys 31:681-687. https://doi .org/ 
10.1016/j.medengphy . 2009.01.003

23. Amabile C, Pillet H, Lafage V, et al (2016) A new quasi-invariant parameter characterizing the postural alignment of young asymptomatic adults. Eur Spine J 25:3666-3674. https : / doi .org/10 .1007/s00586-016-4552-y

24. Coe D (2009) Fisher matrices and confidence ellipses: a quick-start guide and software. arXiv:09064123

25. Conover WJ, Iman RL (1982) Analysis of Covariance Using the Rank Transformation. Biometrics 38:715-724. https://doi.org/10.2307/ 2530051

26. Zaina F, Donzelli S, Lusini M, Negrini S (2012) Correlation between in-brace radiographic correction and short time brace results. Scoliosis 7:1. https://doi .org/10.1186/1748-7161-7-s1-027

27. Clin J, Aubin C-É, Sangole A, et al (2010) Correlation between immediate in-brace correction and biomechanical effectiveness of brace treatment in adolescent idiopathic scoliosis. Spine (Phila Pa 1976) 35:1706-1713. https://doi .org/10.1097/BRS .0b013e3181cb46f6

28. Rigo M, Negrini S, Weiss HR, et al (2006) "SOSORT consensus paper on brace action: TLSO biomechanics of correction (investigating the rationale for force vector selection)." Scoliosis 1:11. https://doi.org/10.1186/ $1748-7161-1-11$

29. Vital JM, Senegas J (1986) Anatomical bases of the study of the constraints to which the cervical spine is subject in the sagittal plane A study of the center of gravity of the head. Surg Radiol Anat 8:169-173. https://doi.org/10.1007/BF02427845

30. Dubousset J (2011) Reflections of an orthopaedic surgeon on patient care and research into the condition of scoliosis. J Pediatr Orthop 31:S1-8. https://doi.org/10.1097/BP0.0b013e3181f73beb

31. Amabile C, Le Huec J-C, Skalli W (2018) Invariance of head-pelvis alignment and compensatory mechanisms for asymptomatic adults older than 49 years. Eur Spine J 27:458-466.

https://doi.org/10.1007/s00586-016-4830-8

32. Guo J, Liu Z, Lv F, et al (2012) Pelvic tilt and trunk inclination: new predictive factors in curve progression during the Milwaukee bracing for adolescent idiopathic scoliosis. Eur Spine J 21:2050-2058. https://doi . org/10.1007/s00586-012-2409-6 